\title{
Comparing space+time decompositions in the post-Newtonian limit
}

\author{
Barak Kol ${ }^{a}$, Michele Levi ${ }^{a}$ and Michael Smolkin ${ }^{b}$ \\ ${ }^{a}$ Racah Institute of Physics, Hebrew University, Jerusalem 91904, Israel \\ barak_kol,michele@phys.huji.ac.il \\ ${ }^{b}$ Perimeter Institute for Theoretical Physics, Waterloo, Ontario N2L 2Y5, Canada \\ msmolkin@perimeterinstitute.ca
}

\begin{abstract}
The relationship between the Arnowitt-Deser-Misner (ADM) field decomposition and the non-relativistic gravitational (NRG) fields attracted considerable interest recently. This paper compares the two, especially with respect to computing the two-body post-Newtonian (PN) effective action within the effective field theory (EFT) approach. Both are space+time decompositions and hence do better than using the standard metric. However, ADM is essentially a reduction over space whereas NRG is essentially a reduction over time. We use a variant of ADM which is linearly equivalent to NRG and the two are identical at order $1 \mathrm{PN}$. We compare the two at order $2 \mathrm{PN}$ and find that $\mathrm{ADM}$ requires the computation of an additional Feynman diagram. We argue that the computational excess will further increase at higher orders.
\end{abstract}




\section{Contents}

$\begin{array}{llr}1 & \text { Introduction } & 1\end{array}$

2 Field definition and action 2

3 Feynman rules $\quad 5$

$\begin{array}{lll}4 & \text { Feynman diagrams and their evaluation at 2PN } & 7\end{array}$

$\begin{array}{llr}5 & \text { Discussion } & 8\end{array}$

\section{Introduction}

The effective field theory (EFT) approach to the two-body post-Newtonian (PN) dynamics in general relativity (GR) was put forward in [1], borrowing ideas from effective quantum field theories. It is based on a hierarchy of scales in the problem and essentially replaces the traditional method of dealing with finite-size objects through matched asymptotic expansion by introducing instead effective interactions of point particles with their background. See [2] for early precursors of the EFT approach to GR. The ability of the method to go beyond the state of the art was demonstrated in [3] ${ }^{1}$ by computing for the first time the next-to-leading spin(1)-spin(2) interaction in the effective two-body action.

In the post-Newtonian limit, spacetime is nearly flat and velocities are slow; hence, there is a distinguished time direction and a space+time decomposition is useful. For that purpose non-relativistic gravitational (NRG) fields were introduced in $[7,8]^{2}$ and were used to give what is probably the shortest derivation of the leading post-Newtonian correction, known as the Einstein-Infeld-Hoffmann interaction [10]. Recently, the full Einstein-Hilbert action was obtained in terms of these fields [11]. Related interesting and relatively recent work on PN and/or EFT appeared in [12].

The definition of NRG fields has some similarities with the well-known ArnowittDeser-Misner (ADM) fields [13]. Actually the two definitions are identical in the static limit (where in particular $g_{0 i}=0$ ) and more generally they are linearly equivalent (at least after some modification of ADM as we review below) and therefore the two-body effective action is identical up to order $1 \mathrm{PN}$. This partial similarity attracted considerable interest and raised the question whether the distinction is essential. ${ }^{3}$ The purpose of this paper is

\footnotetext{
${ }^{1}$ Even if imperfectly, since it was missing certain contributions found in [4] using Hamiltonian methods and also found later in [5] to arise from indirect contributions in the EFT method. See [6] for a derivation using NRG fields (to be introduced below).

${ }^{2}$ See [9] for early precursors of the NRG fields.

${ }^{3}$ Private communications.
} 
to explain the differences both from a conceptual point of view and from a practical and computational PN perspective.

While both NRG and ADM represent space+time decomposition, they are nonlinearly inequivalent and there is a marked difference: NRG is essentially a temporal Kaluza-Klein [14] reduction while ADM is essentially a spatial reduction. Since the PN spacetime is nearly stationary, a temporal reduction is conceptually fit. Yet, from a practical point of view it is desirable to compare the two field definitions as they function while computing the effective two-body PN action. We use the harmonic gauge to enable comparison with previous derivations. Due to the 1PN equivalence, we proceed to order $2 \mathrm{PN}$ (see the review [15] and references therein). The $2 \mathrm{PN}$ action was reproduced within the EFT approach in [16] who found the NRG fields to be preferable for their computations.

In this paper, we reproduce the $2 \mathrm{PN}$ effective action using the ADM fields and compare with [16]. In section 2, we present the field definitions and action, in section 3 the Feynman rules, and in section 4 we evaluate the required Feynman diagrams. Finally our summary and discussions are presented in section 5 .

\section{Field definition and action}

Field definition. The non-relativistic gravitational (NRG) fields were introduced in $[7,8]$ through a temporal Kaluza-Klein [14] reduction followed by a Weyl rescaling

$$
d s^{2}=e^{2 \hat{\phi}}\left(d t-\hat{A}_{i} d x^{i}\right)^{2}-e^{-2 \hat{\phi} / \tilde{d}} \hat{\gamma}_{i j} d x^{i} d x^{j}
$$

where for greater generality we work with an arbitrary spacetime dimension and we denote

$$
\tilde{d}:=d-3 .
$$

Here the NRG fields are hatted to distinguish them from the ADM fields below. The temporal reduction divides the metric into a scalar, a vector and a tensor with respect to spatial transformations, while time shifts gauge the vector. The Weyl rescaling leading to $\hat{\gamma}_{i j}$ is performed to decouple the quadratic action and hence the associated propagators (equivalently, $\hat{\gamma}_{i j}$ appears in the action in a canonical Einstein-Hilbert form).

The utility of the NRG fields received strong support from [16] who reproduced the 2PN effective action through the EFT approach choosing to work with NRG fields. They found essentially two advantages for NRG fields over the standard metric: the quadratic decoupling (this is especially useful since at leading order a compact object couples only to $\hat{\phi}$ ), and the elimination of certain bulk vertices.

The standard ADM decomposition [13] (see also the review [17] and references therein) is given by

$$
d s^{2}=\alpha^{2} d t^{2}-\widetilde{\gamma}_{i j}\left(d x^{i}+\beta^{i} d t\right)\left(d x^{j}+\beta^{j} d t\right),
$$

where $\alpha$ is the lapse and $\beta^{i}$ is the shift vector. It is designed for the initial value problem or time evolution. Actually one notices that in ADM, the shift is on the spatial coordinates, while in standard KK it is on the reduced coordinate, for instance, time in NRG (2.1). In this sense, ADM is nothing but a (Kaluza-Klein) reduction over the spatial directions. 
From this perspective of reducing over space and concentrating on the time dynamics, the ADM fields have the following transformation properties: $\widetilde{\gamma}_{i j}$ is a matrix of temporal scalars, the shift $\beta^{i}$ is a set of temporal vectors $\sim\left(h_{t}\right)^{i}$, and $\alpha^{2}$ is the temporal metric $\sim h_{t t}$. Being 1D, the (temporal) vectors and tensor $\left(\beta^{i}\right.$ and $\left.\alpha^{2}\right)$ are non-dynamic during time evolution. The last statement can be both familiar and surprising from the point of view of numerical relativity. Familiar because given geometrical and source-free initial conditions on a Cauchy surface the lapse and shift are indeed non-dynamic and can be chosen at will (gauge choice). On the other hand, it could be surprising because in the presence of material bodies, the fields including the lapse and shift are determined (in a specified gauge, say harmonic) by elliptic equations with material sources and are not free to choose. The resolution of this tension is to remember that in the latter case, the dynamic degrees of freedom are actually with the material bodies and the gravitational field merely reacts to it, at least in the near zone where radiation is unimportant.

Standard ADM has two clear though fixable drawbacks from the PN perspective. These are a non-flat kinetic metric for $\alpha$ (namely the $\alpha$ dependent prefactor in the kinetic term $\left.S \supset \int \alpha^{-2}|\partial \alpha|^{2}\right)$, and a mix between $\alpha$ and $\widetilde{\gamma}:=\operatorname{tr}\left(\widetilde{\gamma}_{i j}\right)$ at the quadratic level of the form $S \supset \partial \alpha \partial \widetilde{\gamma}$. Both issues produce extra diagrams already at order 1PN and the problem only aggravates with increasing $\mathrm{PN}$ order: the $\alpha$ kinetic term produces a triple vertex for $\alpha$ which contributes starting with a Y-shaped diagram at 1PN, while the mix adds a 2-vertex which appears at $1 \mathrm{PN}$ through the diagram which describes an exchange of $\alpha$ which transforms into $\widetilde{\gamma}$ and back into $\alpha$. However, these drawbacks can be fixed by a "modified ADM decomposition" which we proceed to define, in order to make a more essential and elaborate comparison with the NRG decomposition.

We define a modified ADM decomposition as follows

$$
d s^{2}=e^{2 \phi} d t^{2}-e^{-2 \phi / \tilde{d}} \gamma_{i j}\left(d x^{i}+A^{i} d t\right)\left(d x^{j}+A^{j} d t\right) .
$$

This is obtained from the standard definition in equation (2.3) by defining $\phi:=\log (\alpha)$ in order to have a flat kinetic metric for the scalar (namely $S \supset \int|\partial \phi|^{2}$ rather than $\left.S \supset \int \alpha^{-2}|\partial \alpha|^{2}\right)$; a Weyl rescaled $\gamma_{i j}:=\exp (2 \phi / \tilde{d}) \widetilde{\gamma}_{i j}$ as before to achieve decoupling in the quadratic action; and finally a change of notation $A^{i}:=\beta^{i}$ to make the ADM and NRG notations similar.

Comparing the definitions of the modified ADM from equation (2.4) and the NRG fields in equation (2.1), we observe that they are similar in two ways. First, for $\hat{A}_{i}=A^{i}=0$ they are identical. Second, linearizing around flat space we define

$$
\sigma_{i j}=\gamma_{i j}-\delta_{i j}
$$

and similarly for NRG fields $\hat{\sigma}_{i j}=\hat{\gamma}_{i j}-\delta_{i j}$, and then the two sets are linearly equivalent around flat space, namely $\hat{\phi}=\phi+\ldots, \hat{A}_{i}=A_{i}+\ldots, \hat{\sigma}_{i j}=\sigma_{i j}+\ldots$ where the ellipsis denote terms which are quadratic or higher in the perturbation fields $\phi, A_{i}, \sigma_{i j}$ (the spatial indices $i, j, \ldots$ are raised and lowered here with $\delta_{i j}$ ).

Action. The total action is a sum of three parts

$$
S_{t o t}=S_{E H}+S_{G F}+S_{p},
$$


where $S_{E H}$ is the bulk Einstein-Hilbert action, $S_{G F}$ is the harmonic gauge-fixing term and $S_{p}$ is the particle action.

In order to obtain the Einstein-Hilbert action $S=-1 /(16 \pi G) \int R d V$ we first computed it for the original ADM form of equation (2.3) and then performed a field redefinition. For the first step, we used the non-orthonormal frame method (see for example [11]) and found the well-known result

$$
S_{E H}=-\frac{1}{16 \pi G} \int \alpha \sqrt{\tilde{\gamma}} d^{d-1} x d t\left\{-\left(\left|K_{i j}\right|^{2}-K^{2}\right)-R[\widetilde{\gamma}]\right\},
$$

where the extrinsic curvature is defined by

$$
K_{i j}=-\frac{1}{2} \alpha^{-1}\left(D_{t} \widetilde{\gamma}_{i j}-\widetilde{\gamma}_{k(j} \partial_{i)} A^{k}\right) \equiv-\frac{1}{2} \alpha^{-1}\left(\dot{\widetilde{\gamma}}_{i j}-\mathcal{L}_{A} \widetilde{\gamma}_{i j}\right)
$$

and where

$$
D_{t}:=\partial_{t}-A^{i} \partial_{i}
$$

and $\mathcal{L}_{\mathcal{A}}$ denotes the Lie derivative with respect to $A^{i}$. In addition, we define

$$
\begin{aligned}
K & :=\widetilde{\gamma}^{i j} K_{i j}, \\
\left|K_{i j}\right|^{2} & :=K_{i j} K_{k l} \widetilde{\gamma}^{i k} \widetilde{\gamma}^{j l} .
\end{aligned}
$$

In terms of the modified ADM fields in equation (2.4), one finds (note that this time the new $\gamma_{i j}$ appears, not the pre-Weyl $\widetilde{\gamma}_{i j}$ )

$$
\begin{aligned}
S_{E H}= & -\frac{1}{16 \pi G} \int \sqrt{\gamma} d^{d-1} x d t \\
& \left\{\left(1+\frac{1}{\tilde{d}}\right)\left|\partial_{i} \phi\right|^{2}-R[\gamma]-e^{-2 \phi / \tilde{d}}\left(\left|K_{i j}[\gamma]\right|^{2}-K^{2}[\gamma]\right)\right. \\
& \left.+\left(1+\frac{1}{\tilde{d}}\right) e^{-2(1+1 / \tilde{d}) \phi} D_{t} \phi\left(2 e^{\phi} K[\gamma]+\left(1+\frac{2}{\tilde{d}}\right) D_{t} \phi\right)\right\} .
\end{aligned}
$$

The harmonic gauge-fixing term is defined by

$$
S_{G F}=\frac{1}{2 \cdot 16 \pi G} \int e^{-2 \phi / \tilde{d}} \sqrt{\gamma} d^{d-1} x d t \quad g^{a b} \Gamma_{a} \Gamma_{b},
$$

where $g^{a b} \Gamma_{a} \Gamma_{b}=\Gamma_{\hat{0}}^{2}-e^{2 \phi / \tilde{d}} \gamma^{i j} \Gamma_{\hat{i}} \Gamma_{\hat{j}}$ and $\hat{0}, \hat{i}$ are frame indices. In ADM variables, we find

$$
\begin{aligned}
& \Gamma_{\hat{0}}=e^{-\phi} \partial_{i} A^{i}-\frac{1}{2} e^{-\phi} D_{t} \log \gamma+2\left(1+\frac{1}{\tilde{d}}\right) e^{-\phi} D_{t} \phi, \\
& \Gamma_{\hat{i}}=\Gamma_{i}[\gamma]-e^{-2(1+1 / \tilde{d}) \phi} \gamma_{i j} D_{t} A^{j},
\end{aligned}
$$

where $\Gamma_{i}[\gamma]=\gamma^{j k}\left(\partial_{j} \gamma_{i k}-\partial_{i} \gamma_{j k} / 2\right)$, namely it is the contraction of the Christoffel symbols for the metric $\gamma_{i j}$ which would be used to define the standard harmonic gauge for $\gamma_{i j}$.

The worldline action is approximated at leading EFT order by a point particle action and can be read from equation (2.4) to be

$$
S_{p} \equiv-m \int d \tau=-m \int d t \sqrt{e^{2 \phi}-e^{-2 \phi / \tilde{d}} \gamma_{i j}\left(v^{i}+A^{i}\right)\left(v^{j}+A^{j}\right)},
$$

where we denote the velocity by $v^{i}:=d x^{i} / d t$. 


\section{Feynman rules}

The total action for the modified ADM fields of equation (2.4) is given by equations (2.6) and (2.11-2.14) and we work in 4D for definiteness. From it, we read the Feynman rules. The propagators are

$$
\begin{aligned}
& =\left\langle\phi\left(x_{1}\right) \phi\left(x_{2}\right)\right\rangle=4 \pi G \quad \int_{\mathbf{k}} \frac{e^{i \mathbf{k} \cdot\left(\mathbf{x}_{1}-\mathbf{x}_{2}\right)}}{\mathbf{k}^{2}} \delta\left(t_{1}-t_{2}\right), \\
----\cdot= & =\left\langle A_{i}\left(x_{1}\right) A_{j}\left(x_{2}\right)\right\rangle=-16 \pi G \delta_{i j} \int_{\mathbf{k}} \frac{e^{i \mathbf{k} \cdot\left(\mathbf{x}_{1}-\mathbf{x}_{2}\right)}}{\mathbf{k}^{2}} \delta\left(t_{1}-t_{2}\right), \\
\overline{\overline{-}} & =\left\langle\sigma_{i j}\left(x_{1}\right) \sigma_{k l}\left(x_{2}\right)\right\rangle=32 \pi G P_{i j ; k l} \int_{\mathbf{k}} \frac{e^{i \mathbf{k} \cdot\left(\mathbf{x}_{1}-\mathbf{x}_{2}\right)}}{\mathbf{k}^{2}} \delta\left(t_{1}-t_{2}\right),
\end{aligned}
$$

where $\int_{\mathbf{k}} \equiv \int \frac{d^{3} \mathbf{k}}{(2 \pi)^{3}}$ for abbreviation, and $P_{i j ; k l} \equiv \frac{1}{2}\left(\delta_{i k} \delta_{j l}+\delta_{i l} \delta_{j k}-2 \delta_{i j} \delta_{k l}\right)$. Here and henceforth, the Feynman rules are presented in position space. Note the simple form of the propagators obtained through the exponentiation and Weyl rescaling in the modified definition in equation (2.4), especially for the $\phi$ and $A_{i}$ fields, which dominate in the gravitational interaction. Note also that factors of $16 \pi G$ could have been eliminated from the bulk Feynman rules either by working with units such that $16 \pi G=1$ (or some other constant) or alternatively by computing $16 \pi G S_{\text {eff }}$ rather than $S_{\text {eff }}$.

In addition, there are time-dependent quadratic vertices. Mixed vertices are eliminated by the Weyl rescaling together with the gauge-fixing term. The Feynman rules for quadratic vertices required up to order $2 \mathrm{PN}$ are

$$
\begin{gathered}
-\times=\frac{1}{8 \pi G} \int d^{4} x\left[\partial_{t} \phi(x)\right]^{2}, \\
--\times--=-\frac{1}{32 \pi G} \int d^{4} x\left[\partial_{t} A_{i}(x)\right]^{2} .
\end{gathered}
$$

The crosses represent bulk vertices that contain two time derivatives. As expected, the Feynman rules up to quadratic order are the same as those for the NRG fields.

The Feynman rules for the three-field bulk vertices required up to order $2 \mathrm{PN}$ are

$$
\begin{aligned}
& =-\frac{1}{8 \pi G} \int d^{4} x\left[\phi(x)\left(\partial_{i} A_{j}(x)\left(\partial_{i} A_{j}(x)+\partial_{j} A_{i}(x)\right)-\left(\partial_{i} A_{i}(x)\right)^{2}\right)\right], \\
& =\frac{1}{16 \pi G} \int d^{4} x\left[2 \sigma_{i j}(x) \partial_{i} \phi(x) \partial_{j} \phi(x)-\sigma_{j j}(x) \partial_{i} \phi(x) \partial_{i} \phi(x)\right], \\
& =-\frac{1}{4 \pi G} \int d^{4} x\left[A_{i}(x) \partial_{i} \phi(x) \partial_{t} \phi(x)\right], \\
& =-\frac{1}{2 \pi G} \int d^{4} x\left[\phi(x)\left(\partial_{t} \phi(x)\right)^{2}\right],
\end{aligned}
$$

where there is no distinction between lower and upper $i, j, \ldots$ indices (or equivalently they are raised and lowered with $\delta_{i j}$ ). 
Note that only the $\phi A^{2}$ vertex in equation (3.6) is different than the respective vertex in terms of the NRG fields. This is consistent with the fact that differences can only occur in vertices which involve the field $A_{i}$. Yet the $A \partial \phi \dot{\phi}$ vertex turns out to be the same.

Now, we consider the gravitational coupling to the massive compact objects. The Feynman rules for the one-field couplings to the worldline mass required up to order $2 \mathrm{PN}$ are

$$
\begin{aligned}
& =-m \int d t \phi(\mathbf{x}(t))\left[1+\frac{3}{2} v(t)^{2}+\frac{7}{8} v(t)^{4}+\cdots\right], \\
& =m \int d t A_{i}(\mathbf{x}(t)) v^{i}(t)\left[1+\frac{1}{2} v(t)^{2}+\cdots\right] \\
& =\frac{m}{2} \int d t \sigma_{i j}(\mathbf{x}(t)) v^{i}(t) v^{j}(t)[1+\cdots]
\end{aligned}
$$

where the heavy solid lines represent the worldlines, and the spherical black vertices represent the masses on the worldline. The ellipsis denotes higher orders in $v$, beyond the order considered here.

The Feynman rules for two-field worldline vertices required up to order $2 \mathrm{PN}$ are

$$
=-\frac{m}{2} \int d t \phi(\mathbf{x}(t))^{2}\left[1-\frac{9}{2} v(t)^{2}+\cdots\right],
$$

Note the appearance of an $A^{2}$ two-field worldline coupling in equation (3.14) already at leading order in $v$ unlike the case for NRG fields, and that the $\phi A$ two-field worldline coupling in equation (3.15) is different as well (larger by a factor of $(-3)$ ). We note again that the differences occur only in couplings which involve the field $A_{i}$.

Finally, the three-field couplings to the worldline required up to order $2 \mathrm{PN}$ are represented by the following Feynman rule:

$$
\left\lceil=-\frac{m}{6} \int d t \phi(\mathbf{x}(t))^{3}[1+\cdots]\right.
$$




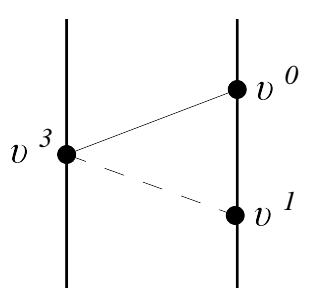

(a)

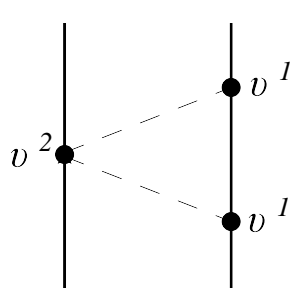

(b)

Figure 1. 2PN Feynman diagrams of two-field exchange including differences from diagrams with NRG fields. (b) An additional diagram that is eliminated when NRG fields are used. These diagrams should be included together with their mirror images.

\section{Feynman diagrams and their evaluation at $2 \mathrm{PN}$}

The differences in the EFT calculation of the binary interaction start to appear at order 2PN (similarly, they appear already in the next-to-leading order of the spin-orbit interaction). That is so because all the Feynman rules required up to 1PN are identical: most rules are identical due to linear equivalence whereas the $\phi^{2}$ worldline coupling from equation (3.13) coincides due to static equivalence (namely for $A^{i}=0$ ). The differences at $2 \mathrm{PN}$ occur only in two topologies (out of the eight topologies contributing at this order): the $\mathrm{V}$ topology of two-field exchange, and the $\mathrm{Y}$ topology of the cubic vertices.

Two-field exchange. Since the $\phi A$ two-field mass coupling in equation (3.15) is different than the respective mass coupling in terms of NRG fields, the respective Feynman diagram, shown in figure 1(a) has a different value, given by

$$
\text { figure } 1(a)=-12 \frac{G^{2} m_{1} m_{2}\left(m_{1}+m_{2}\right)}{r^{2}} \mathbf{v}_{1} \cdot \mathbf{v}_{2},
$$

where here and henceforth a prefactor of $\int d t$ is suppressed and omitted from diagram values. Moreover, the 'new' $A^{2}$ two-field mass coupling in equation (3.14) gives rise to an additional Feynman diagram depicted in figure 1(b), and evaluated to be

$$
\text { figure } 1(b)=8 \frac{G^{2} m_{1} m_{2}}{r^{2}}\left(m_{1} \mathbf{v}_{1}^{2}+m_{2} \mathbf{v}_{2}^{2}\right) \text {. }
$$

Cubic gravitational interaction. Since only the $\phi A^{2}$ vertex in equation (3.6) differs from the respective vertex in terms of NRG fields, the Feynman diagrams including it, shown in figures 2(a), 2(b), have different values given by

$$
\begin{aligned}
& \text { figure } 2(a)=8 \frac{G^{2} m_{1} m_{2}\left(m_{1}+m_{2}\right)}{r^{2}} \mathbf{v}_{1} \cdot \mathbf{v}_{2}, \\
& \text { figure } 2(b)=-4 \frac{G^{2} m_{1} m_{2}}{r^{2}}\left(m_{1} \mathbf{v}_{1}^{2}+m_{2} \mathbf{v}_{2}^{2}\right) .
\end{aligned}
$$

Total effective action at $\mathbf{2 P N}$. Altogether our EFT calculation in terms of ADM-related fields reproduces the total $S_{\text {eff }}$ at $2 \mathrm{PN}$ as found in [16] which used NRG fields (in both 


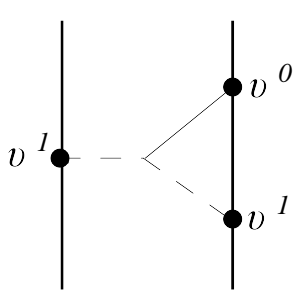

(a)

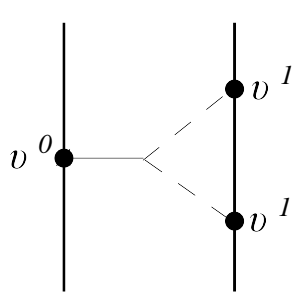

(b)

Figure 2. 2PN Feynman diagrams with a 3-field vertex including differences from diagrams with NRG fields. These diagrams should be included together with their mirror images.

cases, the harmonic gauge was used). The breakdown of this net result is as follows. Only four diagrams are different in the ADM calculation. Diagrams (h) and (k) in [16] (see figure 5 and equations (31), (43) there) yield terms of the same form, with coefficients ${ }^{4} 4$ and (-8), respectively, and a total of (-4). This form appears here in figures 1(a) and 2(a). Figure 1(a) yields the coefficient $(-12)$ (recall that the vertex in equation (3.15) is rescaled by $(-3)$ relative to [16]), and figure 2 (a) yields ${ }^{5}$ the coefficient 8 with the same total of $(-4)$. The diagrams in figures $1(\mathrm{~b})$ and 2(b) also yield terms of the same form with coefficients 8 and (-4), respectively, and a total of 4, whereas in [16] the diagram corresponding to figure 1 (b) here is not present, and figure 2(b) here is replaced (see footnote 7) by diagram (l) (see figure 5 and equation (44) there) and is found to have a coefficient 4, which is identical with our total.

\section{Discussion}

Comparing the computations of the $2 \mathrm{PN}$ effective action using the modified ADM fields with the NRG fields, the Feynman rules are equivalent up to quadratic order in the bulk vertices and linear order in the worldline vertices. They differ for two vertices: the $\phi A$ worldline vertex in equation (3.15) and the bulk $\phi A^{2}$ vertex in equation (3.6). In addition, there is a new $A^{2}$ worldline vertex in equation (3.14). Accordingly altogether, 18 of the diagrams are the same, 3 have different values but comparable computational cost, and the ADM computation requires to evaluate one extra diagram, namely that of figure 1(b), which is factorizable (namely, the computation of the diagram factorizes into a product, and each factor is associated with a sub-diagram). It should be borne in mind that had we not modified the ADM fields (along similar lines of the NRG fields definition), the computational excess would have shown up already at 1PN.

At higher orders. At higher orders, the additional computational cost of the modified ADM fields will further increase due to at least two reasons: first, the extra $A^{2}$ worldline vertex from equation (3.14) will require the computation of several additional diagrams, as can be seen in figure 3. In addition there are other worldline vertices which appear at 3PN

\footnotetext{
${ }^{4}$ Note that [16] compute the potential $V$ which is defined to be minus the value of the diagram.

${ }^{5}$ Recall the different vertex in equation (3.6).
} 


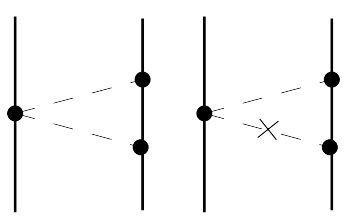

(a1) (a2)

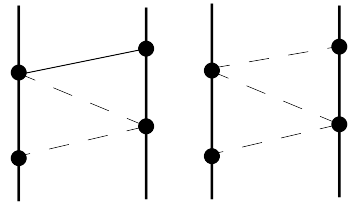

(b1)

(b2)

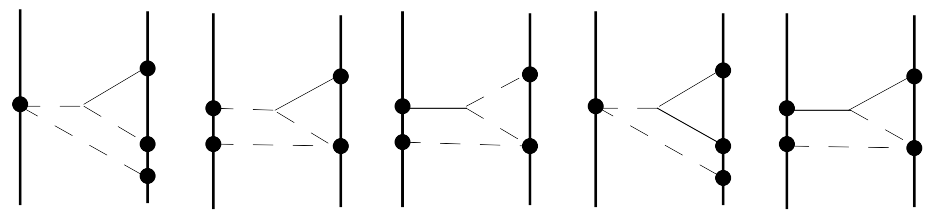

(c2)

(c3)

(c4)

(c5)

Figure 3. Additional Feynman diagrams including the extra 2-field $A^{2}$ worldline vertex, which appear at $3 \mathrm{PN}$ if the modified ADM fields are used. In NRG fields, an $A^{2}$ vertex does not appear altogether at $3 \mathrm{PN}$, but rather it appears first at $4 \mathrm{PN}$ through the term $(\vec{A} \cdot \vec{v})^{2} v^{2}$. Diagrams (a1)-(a2) contribute at order $G^{2}$, while diagrams (b1)-(c5) contribute at order $G^{3}$. Note that while diagram (a1) appears already at $2 \mathrm{PN}$, the velocity dependence of its vertices contributes also to $3 \mathrm{PN}$.

in ADM (but not in NRG), such as a new $\sigma_{i j} A^{i} v^{j}$ worldline vertex. Second, by comparing the ADM bulk action in equations (2.11)-(2.13) and the NRG action in [11] (incorporating the harmonic gauge), one finds that additional bulk vertices will appear or get complicated at higher orders, and we give below several examples.

The following vertices appear at $3 \mathrm{PN}$ and $4 \mathrm{PN}$ in $\mathrm{ADM}$ :

$$
\begin{gathered}
\left.-2 \phi(x)\left(\partial_{t} A_{i}(x)\right)^{2}\right], \\
=-\frac{1}{8 \pi G} \int d^{4} x\left[\phi(x)\left(\partial_{i} A_{j}(x)\left(\partial_{i} A_{j}(x)+\partial_{j} A_{i}(x)\right)-\left(\partial_{i} A_{i}(x)\right)^{2}\right)\right. \\
=\frac{1}{\pi G} \int d^{4} x\left[\phi(x) \partial_{t} \phi(x) \partial_{i} \phi(x) A_{i}(x)\right], \\
=-\frac{1}{32 \pi G} \int d^{4} x\left(A_{i}(x) \partial_{i} A_{j}(x)\right)^{2} .
\end{gathered}
$$

In NRG, the first vertex is stationary and hence simpler, while the quartic vertices are absent altogether.

\section{Acknowledgments}

This research is supported by the Israel Science Foundation grant no 607/05, by the German Israel Cooperation Project grant DIP H.52, and by the Einstein Center at the Hebrew University. Research at Perimeter Institute is supported by the Government of Canada through Industry Canada and by the Province of Ontario through the Ministry of Research \& Innovation. 


\section{References}

[1] W. D. Goldberger and I. Z. Rothstein, Phys. Rev. D 73, 104029 (2006)

W. D. Goldberger, arXiv:hep-ph/0701129

[2] T. Damour and G. Esposito-Farese, Phys. Rev. D 53, 5541 (1996)

[3] R. A. Porto and I. Z. Rothstein, Phys. Rev. Lett. 97, 021101 (2006)

[4] J. Steinhoff, S. Hergt and G. Schaefer, Phys. Rev. D 77, 081501 (2008)

[5] R. A. Porto and I. Z. Rothstein, Phys. Rev. D 78, 044012 (2008) [Erratum-ibid. D 81, $029904(2010)]$

[6] M. Levi, Phys. Rev. D 82, 064029 (2010)

[7] B. Kol and M. Smolkin, Phys. Rev. D 77, 064033 (2008)

[8] B. Kol and M. Smolkin, Class. Quant. Grav. 25, 145011 (2008)

[9] H. Yilmaz, Phys. Rev. 1111417 (1958)

L. Blanchet and T. Damour, Annales Poincare Phys. Theor. 50, 377 (1989)

G. Schaefer, Gen. Rel. Grav. 36, 2223 (2004)

[10] A. Einstein, L. Infeld and B. Hoffmann, Annals Math. 39, 65 (1938)

[11] B. Kol and M. Smolkin, arXiv:1009.1876 [hep-th]

[12] R. A. Porto, A. Ross and I. Z. Rothstein, JCAP 1103, 009 (2011)

M. Levi, Phys. Rev. D 82, 104004 (2010)

D. L. Perrodin, arXiv:1005.0634 [gr-qc]

R. A. Porto, Class. Quant. Grav. 27, 205001 (2010)

D. Brizuela and G. Schaefer, Phys. Rev. D 81, 084014 (2010)

W. D. Goldberger and A. Ross, Phys. Rev. D 81, 124015 (2010)

J. B. Gilmore, A. Ross and M. Smolkin, JHEP 0909, 104 (2009)

C. R. Galley and M. Tiglio, Phys. Rev. D 79, 124027 (2009)

U. Cannella and R. Sturani, Gen. Rel. Grav. 42, 2491 (2010)

V. Cardoso, O. J. C. Dias and P. Figueras, Phys. Rev. D 78, 105010 (2008)

B. Kol, Gen. Rel. Grav. 40, 2061 (2008) [Int. J. Mod. Phys. D 17, 2617 (2009)]

J. Le Witt and S. F. Ross, JHEP 1001, 101 (2010)

R. Emparan, T. Harmark, V. Niarchos and N. A. Obers, JHEP 1003, 063 (2010)

B. Kleihaus, J. Kunz, E. Radu and C. Stelea, JHEP 0909, 025 (2009)

M. Headrick, S. Kitchen and T. Wiseman, Class. Quant. Grav. 27, 035002 (2010)

R. Emparan, T. Harmark, V. Niarchos and N. A. Obers, Phys. Rev. Lett. 102, 191301 (2009)

Y. Z. Chu, Phys. Rev. D 79, 044031 (2009)

[13] R. L. Arnowitt, S. Deser and C. W. Misner, Gravitation: an introduction to current research, Louis Witten ed. (Wiley 1962), pp 227-65

[14] T. Kaluza, Sitzungsber. Preuss. Akad. Wiss. Berlin. (Math. Phys.) 1921966 (1921)

O. Klein, Zeitschrift für Physik A Hadrons and Nuclei 37 12, 895 (1926)

[15] L. Blanchet, Living Rev. Rel. 5, 3 (2002), update: Living Rev. Rel. 9, 4 (2006)

L. Blanchet, arXiv:0907.3596 [gr-qc]

[16] J. B. Gilmore and A. Ross, Phys. Rev. D 78, 124021 (2008)

[17] E. Gourgoulhon, arXiv:gr-qc/0703035 